\title{
A comparison of uni-dimensional and bi-dimensional generalization testing in pigeons'
}

\author{
MARILLA D. SVINICKI and DAVID R. \\ THOMAS, University of Colorado, Boulder, \\ Colo. 80302
}

Three groups of pigeons were trained to peck at a white vertical line on a yellowish-green surround for variable. interval reinforcement and were then tested for generalization, in extinction, along the wavelength dimension (Group $B, N=10$ ), the angularity dimension (Group $C$, $N=10$ ), or along both dimensions presented separately and in alternating sequence (Group $A, N=20$ ). Group A produced both absolute and relative gradients comparable to those obtained from Groups $B$ and $C$. Several prior studies have similarly failed to find successive contextual effects in general ization testing in pigeons.

Kalish \& Haber (1963) trained pigeons to respond to a single spectral wavelength and then tested for generalizations in two ways: (1) with separate groups, each tested in extinction at a single spectral value, and (2) within-Ss, with each animal exposed many times to 11 different test stimuli during extinction. These authors claimed that multistimulus testing sharpened the gradient relative to single-stimulus testing, presumably by providing $S$ s with opportunity to compare the test stimuli. Thomas \& Barker (1964) subsequently pointed out that more extinction was allowed to occur (i.e., the test took longer) in the multistimulus condition, and that this alone would account for the obtained difference in gradient slopes. Hiss \& Thomas (1963) compared single-stimulus and multistimulus tests before measurable extinction had occurred and found no systematic differences between the gradients obtained.

Several other studies have confirmed the finding that generalization gradients obtained within-Ss in extinction (for pigeons) are essentially free of successive contextual effects. Thomas \& Barker (1964) found no evidence for a "central tendency effect," i.e., a tendency for Ss to respond most frequently to stimuli in the center of the series of test stimuli. Friedman (1963) found no "units" effect, i.e., a reduction in responding as a function of the number of intervening generalization test stimuli. Both of these effects had been obtained in wavelength generalization studies with humans (cf. Thomas \& Jones, 1962; Thomas \& Hiss, 1963).
The present study inquired whether the successive presentation of stimuli from wavelength and angularity dimensions would alter the nature of the gradients obtained, in comparison with unidimensional testing. This question is of practical significance because investigators are concerning themselves with increasing frequency with generalization along several test dimensions (cf. Butter, 1963; Cross, 1965; etc.). ${ }^{2}$ Typically the Ss have been tested along different dimensions in a single test session without concern for the possible confounding effects of this procedure. This experiment was designed to determine whether such a concern is warranted.

\section{SUBJECTS}

Forty experimentally naive domestic pigeons, maintained at approximately $75 \%$ of their free-feeding weights, were used.

\section{APPARATUS}

Two Grason-Stadler pigeon test chambers, described in detail by Thomas, Freeman, Svinicki, Burr, \& Lyons (in press), were used.

\section{PROCEDURE}

On the first day, all Ss were magazine trained and the key-peck response was conditioned to a blank white key. On the second day and thereafter the stimulus was a vertical white line on a yellowish-green surround $(555 \mathrm{~nm})$ and each $S$ received 30 reinforcements on a continuous reinforcement schedule (CRF). After two more days of training on CRF, reinforcements were earned with decreasing frequency over a period of several days until a variableinterval 1 -min schedule was in effect. Ten days of training on this schedule followed. Then, after a 5-min warm-up under the usual training condition, the Ss were tested, in extinction, for generalization along the wavelength and/or line angle dimensions. Group A (composite test group) was tested along both dimensions. The test consisted of alternate $30-\mathrm{sec}$ presentations of various wavelengths of light $(501 \mathrm{~nm}, 538 \mathrm{~nm}$, $555 \mathrm{~nm}, 576 \mathrm{~nm}$, and $606 \mathrm{~nm}$ ), with no line superimposed, and various line angles $(30$, $60,90,120$, and $150 \mathrm{deg}$ ), each on a black surround. No blackouts or no-stimulus periods intervened between stimulus presentations. The stimuli were presented in 9 blocks of 10 stimuli, each stimulus occurring once in each block. The two dimensions were regularly alternated, but order of presentation within a dimension was random. Group B was tested for generaliza- tion along the wavelength dimension only. These Ss received 30-sec presentations of the five wavelengths (with noline present) in 18 blocks, each stimulus occurring once in each block in random order. Thus the total number of stimulus presentations in the generalization test was the same for Group B as for Group A. Group C was tested in the same manner as Group B, but along the line angle dimension, with the white lines presented on a black surround.

\section{RESULTS AND DISCUSSION}

For each $S$ a record was kept of the total number of responses made to each of the generalization test stimuli. For Ss in Group A, separate gradients were thus obtained along both the wavelength and the angularity dimension. A relative generalization gradient was also computed, in terms of the proportion of total responses (within a dimension) emitted to each of the test stimuli. Absolute and relative gradients were also obtained from Groups B and C. Since these groups had twice as many presentations of each test stimulus as did the $\mathrm{Ss}$ in Group A, the totals obtained from these Ss were divided in half.

In Fig. 1 are presented the mean absolute wavelength gradients of Groups A and B. The two gradients are very close to parallel, with the Group A gradient slightly higher. An analysis of variance of these data reveals that the group effect and the Group by Stimuli interaction do not approach significance $(F<1)$. Figure 2 shows that the relative wavelength gradients of these two groups are virtually identical, and analysis of variance confirms this $(F<1$ for both group and interaction effects).

In Fig. 3 are presented the mean absolute angularity generalization gradients of Groups $A$ and $C$. The two gradients are quite similar, with a slight suggestion that the Group A (composite test) gradient is flatter.

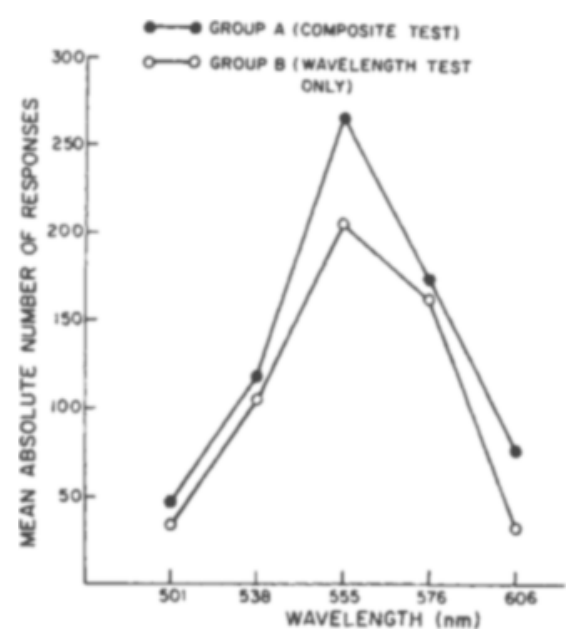

Fig. 1. Mean absolute wavelength general ization gradients of Groups $A$ and $B$. 


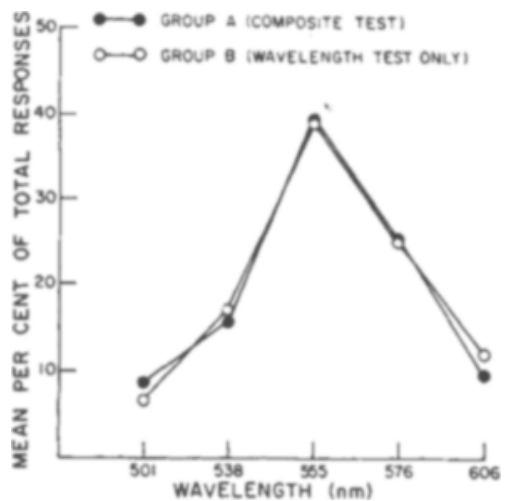

Fig. 2. Mean relative wavelength generalization gradients of Groups $A$ and $B$.

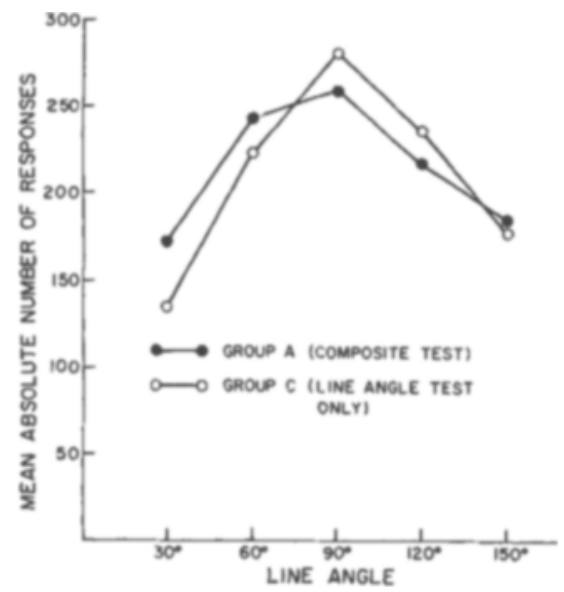

Fig. 3. Mean absolute angularity generalizationgradients of Groups A and C.

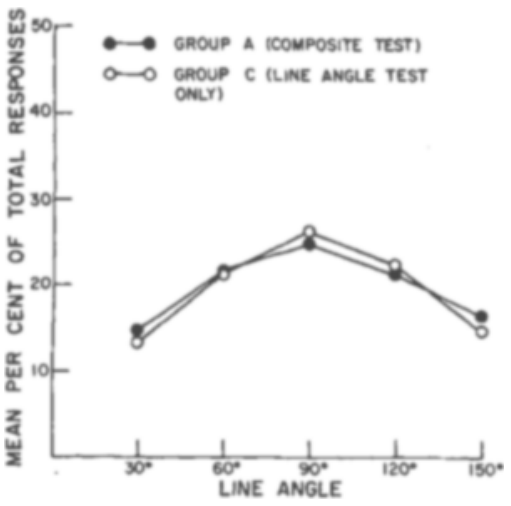

Fig. 4. Mean relative angularity generalization gradients of Groups $A$ and $C$.

Neither group nor interaction effects approach statistical significance, however [group, $\mathrm{F}<1$; interaction, $\mathrm{F}(4,112)=1.7$, $\mathrm{p}>.10]$. The mean relative angularity gradients of Groups $A$ and $C$, shown in Fig. 4, are virtually identical, and again statistical analysis reveals no significant group or Group by Stimuli interaction effect $(\mathrm{F}<1)$.

This experiment agrees with the earlier ones performed with pigeons in finding that, following variable-interval training, the generalization gradient obtained within-Ss in extinction is substantially immune from successive contextual effects. This is the case whether the gradient is plotted in absolute or in relative terms. There is a slight suggestion from the data of the present study that the alternated presence of wavelength stimuli in the test may flatten the angularity gradient, but the effect falls far short of significance despite the fact that larger groups were used in this study than is typical of operant stimulus control studies. The failure to reject the null hypothesis cannot be taken to mean that there is absolutely no effect of having two stimulus dimensions present during generalization testing, but it can certainly be said that any effect obtained is likely to be of very limited magnitude and thus of no practical consequence. In view of the consistent demonstration of the insensitivity of the generalization gradient (yielded by these procedures) to successive contextual effects, the increased efficiency afforded by within-Ss designs in the study of bidimensional stimulus generalization adequately justifies their use.

\section{REFERENCES}

BUTTER, C. M. Stimulus generalization along one and two dimensions in pigeons. Journal of Experimental Psychology, 1963, 65, 339-346. CROSS, D. V. Metric properties of multidimensional stimulus generalization. In D. I.
Mostofsky (Ed.), Stimulus generalization. Stanford, Calif.: Stanford University Press, 1965. Pp. 72-93.

FRIEDMAN, H. Wavelength generalization as a function of spacing of test stimuli. Journal of Experimental Psychology, 1963, 65, 334-338.

HISS, R. H., \& THOMAS, D. R. Stimulus generalization as a function of testing procedure and response measure. Journal of Experimental Psychology, 1963, 65, 587-592.

KALISH, H. I., \& HABER, A. Generalization: I. Generalization gradients from single and multiple stimulus points. II. Generalization of inhibition. Journal of Experimental Psychology, 1963, 65, 176-181.

THOMAS, D. R., \& BARKER, E. G. The effects of extinction and "central tendency" on stimulus generalization in pigeons. Psychonomic Science, 1964, 1, 119-120.

THOMAS, D. R., \& HISS, R. H. A test of the "Units Hypothesis" employing wave-length generalization in human subjects. Journal of Experimental Psychology, 1963, 65, 59-62.

THOMAS, D. R., \& JONES, C. G. Stimulus generalization as a function of the frame of reference. Journal of Experimental Psychology, 1962,64, 77-80.

THOMAS, D. R., F'REEMAN, F., SVINICKI, J.G. BURR, D. E. S., \& LYONS, J. The effects of extra-dimensional training on stimulus generalization. Journal of Experimental Psy chology, in press.

\section{NOTES}

1. This research was supported by Research Grant HD-03486 and Training Grant MH-10427 under the direction of the second author. D. E. Scott Burr and Robert Hintz aided in the collection of the data.

2. Thomas, D. R., Burr, D. E. S., \& Svinicki, M. $D$. Evidence for a positive relationship between degree of control acquired by two dimensions of a complex stimulus. Submitted to Nature, 1969.

\section{Chronic effects of social stimuli on adrenocortical function in male mice'}

\section{JOHN ARCHER, ${ }^{2}$ University of Bristol, England}

Recent work showed that when previously isolated male mice are housed in proximity with one another, their adrenals become heavier than those of mice remaining in isolation. The present experiment revealed that this adrenal hypertrophy no longer occurred when the differential housing period was longer, i.e., 9 weeks instead of 2 weeks. These results suggest that the adrenal response was associated with the reaction of strange animals to one another, rather than the chronic effects of social stimulation.
Experiments carried out on rodents reveal that isolation and group housing exert contrasting effects on adrenocortical function. The precise nature of these effects seems to depend on the experimental procedure used: Preexperimental isolation and the occurrence of aggressive behavior are associated with higher levels of adrenal function in the grouped animals ("social stress"), and preexperimental grouping and the absence of aggression are often associated with smaller differences in the opposite direction ("isolation stress"). The literature on which the above conclusions are based will be considered more fully in a review article, but typical examples of social stress are to be found in the work of 